\title{
Nonlinear nonhomogeneous Dirichlet problems with singular and convection terms
}

\author{
Nikolaos S. Papageorgiou' and Youpei Zhang ${ }^{2,3 *}$
}

\author{
"Correspondence: \\ zhangypzn@163.com; \\ youpei.zhang@inf.ucv.ro \\ ${ }^{2}$ School of Mathematics and \\ Statistics, Central South University, \\ Changsha, Hunan 410083, China \\ ${ }^{3}$ Department of Mathematics, \\ University of Craiova, Street A.I. Cuza \\ No. 13, 200585 Craiova, Romania \\ Full list of author information is \\ available at the end of the article
}

\begin{abstract}
We consider a nonlinear Dirichlet problem driven by a general nonhomogeneous differential operator and with a reaction exhibiting the combined effects of a parametric singular term plus a Carathéodory perturbation $f(z, x, y)$ which is only locally defined in $x \in \mathbb{R}$. Using the frozen variable method, we prove the existence of a positive smooth solution, when the parameter is small.
\end{abstract}

MSC: 35B50; 35J75; 35J92

Keywords: Frozen variable method; Nonlinear regularity; Minimal positive solution; Leray-Schauder alternative principle; Truncation; Fixed point; Convection term

\section{Introduction}

Let $\Omega \subseteq \mathbb{R}^{N}$ be a bounded domain with $C^{2}$-boundary $\partial \Omega$. In this paper we study the following nonhomogeneous parametric singular Dirichlet problem with gradient dependence (convection):

$$
\left\{\begin{array}{l}
-\operatorname{div} a(D u(z))=\lambda u(z)^{-\eta}+f(z, u(z), D u(z)) \quad \text { in } \Omega, \\
\left.u\right|_{\partial \Omega}=0, \quad u>0, \quad \lambda>0, \quad 0<\eta<1 .
\end{array}\right\}
$$

In this problem, the map $a: \mathbb{R}^{N} \mapsto \mathbb{R}^{N}$ in the differential operator is continuous and strictly monotone (thus, maximal monotone too) and satisfies certain other growth and regularity conditions listed in hypotheses $\mathrm{H}_{0}$ below (see Sect. 2). These conditions are general and provide a broad framework in which we can fit many differential operators of interest, such as the $p$-Laplacian and the $(p, q)$-Laplacian (that is, the sum of a $p$-Laplacian and of $q$-Laplacian). In the reaction (right-hand side) of $\left(P_{\lambda}\right)$, we have the competing effects of a parametric singular term $u \mapsto \lambda u^{-\eta}$ with $\lambda>0$ being the parameter and of a perturbation $u \mapsto f(z, u, D u)$ which is a Carathéodory function (that is, for all $x \in \mathbb{R}, y \in \mathbb{R}^{N}$ the function $z \mapsto f(z, x, y)$ is measurable and for a.a. $z \in \Omega,(x, y) \mapsto f(z, x, y)$ is continuous). So, this perturbation is gradient dependent and on $f(z, \cdot, y)$ we do not impose any growth condition. Instead we assume that near zero the function $x \mapsto f(z, x, y)$ exhibits a kind of oscillatory behavior.

The gradient dependence of the reaction means that problem $\left(P_{\lambda}\right)$ is not variational and so eventually our method of proof is going to be topological. We use the so-called

(c) The Author(s) 2020. This article is licensed under a Creative Commons Attribution 4.0 International License, which permits use, sharing, adaptation, distribution and reproduction in any medium or format, as long as you give appropriate credit to the original author(s) and the source, provide a link to the Creative Commons licence, and indicate if changes were made. The images or other third party material in this article are included in the article's Creative Commons licence, unless indicated otherwise in a credit line to the material. If material is not included in the article's Creative Commons licence and your intended use is not permitted by statutory regulation or exceeds the permitted use, you will need to obtain permission directly from the copyright holder. To view a copy of this licence, visit http://creativecommons.org/licenses/by/4.0/. 
"frozen variable method". According to this approach, in the perturbation $f(z, x, y)$, we fix ("freeze") the $y$-variable. This leads to a variational problem, which a priori can be solved using tools from the critical point theory. However, the presence of the singular term leads to an energy functional which is not $C^{1}$ and so we have difficulty in applying the minimax theorems of critical point theory. So, we need to find a way to bypass the singularity and deal with $C^{1}$-functionals. This is done by considering the purely singular problem (that is, $f \equiv 0$ ), which we show that for every $\lambda>0$ it has a unique positive smooth solution which converges to zero in $C_{0}^{1}(\bar{\Omega})$ as $\lambda \rightarrow 0^{+}$. We use this solution and its properties and truncation techniques, to show that, for all small values of the parameter $\lambda>0$, the "frozen problem" has at least one positive smooth solution. In order to use topological tools (fixed point theory), we need to find a canonical way to choose such a positive solution. This is done by showing that the frozen problem has a smallest positive solution (minimal positive solution). Then we show that the minimal solution map satisfies all the requirements of Leray-Schauder alternative principle (see Sect. 2) and so we can produce a positive solution for $\left(P_{\lambda}\right)$ when $\lambda>0$ is small.

Recently there have been published some existence results for nonlinear problem with convection. We mention the work of Bai [1], Bai-Gasinski-Papageorgiou [2], Candito-Gasinski-Papageorgiou [4], Faraci-Motreanu-Puglisi [5], Gasinski-KrechPapageorgiou [6], Gasinski-Papageorgiou [10], Gasinski-Winkert [11], Liu-Papageorgiou [18], Papageorgiou-Rădulescu-Repovš [20], Papageorgiou-Vetro-Vetro [24], Tanaka [31]. However, none of the aforementioned works involves singular terms. The only work examining the combined effects of singular and convection terms, is the recent paper of Papageorgiou-Rădulescu-Repovš [22], which deals with a nonparametric Neumann problem driven by the $p$-Laplacian. Nonlinear singular Dirichlet problems were also investigated in the paper of Papageorgiou-Winkert [25] for different settings and conditions.

\section{Mathematical background-hypotheses}

The main spaces in the analysis of our problem $\left(P_{\lambda}\right)$, are the Sobolev space $W_{0}^{1, p}(\Omega)$ and the Banach space $C_{0}^{1}(\bar{\Omega})=\left\{u \in C^{1}(\bar{\Omega}):\left.u\right|_{\partial \Omega}=0\right\}$. By $\|\cdot\|$ we denote the norm of the Sobolev space $W_{0}^{1, p}(\Omega)$. On account of the Poincaré inequality, we have

$$
\|u\|=\|D u\|_{p} \quad \text { for all } u \in W_{0}^{1, p}(\Omega)
$$

The Banach space $C_{0}^{1}(\bar{\Omega})$ is an ordered Banach space with positive (order) cone $C_{+}=$ $\left\{u \in C_{0}^{1}(\bar{\Omega}): u(z) \geq 0\right.$ for all $\left.z \in \bar{\Omega}\right\}$. This cone has a nonempty interior given by

$$
\operatorname{int} C_{+}=\left\{u \in C_{+}: u(z)>0 \text { for all } z \in \Omega,\left.\frac{\partial u}{\partial n}\right|_{\partial \Omega}<0\right\} \text {, }
$$

with $n(\cdot)$ being the outward unit normal on $\partial \Omega$.

Let $X$ be a Banach space and $\xi: X \mapsto X$. We say that $\xi(\cdot)$ is "compact" if it is continuous and for every $B \subseteq X$ bounded, the set $\overline{\xi(B)} \subseteq X$ is compact. The "Leray-Schauder alternative principle" asserts the following.

Theorem 1 If $X$ is a Banach space, $\xi: X \mapsto X$ is compact and

$$
\mathcal{D}(\xi)=\{u \in X: u=t \xi(u) \text { for some } 0<t<1\}
$$


then the following alternative holds:

(a) $\mathcal{D}(\xi)$ is unbounded; or

(b) $\xi(\cdot)$ has a fixed point.

Let $\beta \in C^{1}(0, \infty)$ with $\beta(t)>0$ for all $t>0$ and assume that

$$
\begin{aligned}
& 0<\hat{c} \leq \frac{\beta^{\prime}(t) t}{\beta(t)} \leq c_{0}, \\
& c_{1} t^{p-1} \leq \beta(t) \leq c_{2}\left(t^{s-1}+t^{p-1}\right),
\end{aligned}
$$

for all $t>0$, some $1 \leq s<p<N, 0<c_{1}<c_{2}$.

Then our hypotheses on the map $a(\cdot)$ are the following.

$\mathrm{H}_{0}: a(y)=a_{0}(|y|) y$ for all $y \in \mathbb{R}^{N}$, with $a_{0}(t)>0$ for all $t>0$ and

(i) $a_{0} \in C^{1}(0, \infty), t \mapsto a_{0}(t) t$ is strictly increasing on $(0, \infty), a_{0}(t) t \rightarrow 0^{+}$as $t \rightarrow 0^{+}$and $\lim _{t \rightarrow 0^{+}} \frac{a_{0}^{\prime}(t) t}{a_{0}(t)}>-1$;

(ii) there exists $c_{3}>0$ such that

$$
|\nabla a(y)| \leq c_{3} \frac{\beta(|y|)}{|y|} \quad \text { for all } y \in \mathbb{R}^{N} \backslash\{0\}
$$

(iii) $\frac{\beta(|y|)}{|y|}|\xi|^{2} \leq(\nabla a(y) \xi, \xi)_{\mathbb{R}^{N}}$ for all $y \in \mathbb{R}^{N} \backslash\{0\}$, all $\xi \in \mathbb{R}^{N}$;

(iv) if $G_{0}(t)=\int_{0}^{t} a_{0}(s) s d s, t \geq 0$, then there exist $q \in(1, p)$ and $c_{*}>0$ such that

$$
\limsup _{t \rightarrow 0^{+}} \frac{q G_{0}(t)}{t^{q}} \leq c_{*}
$$

Remark 1 Such conditions on the differential operator were used in the context of singular or convection problems, also by Papageorgiou-Rădulescu-Repovš [23] and CanditoGasinski-Papageorgiou [4]. Hypotheses $\mathrm{H}_{0}$ (i), (ii), (iii) are motivated by the nonlinear regularity theory of Lieberman [17] and the nonlinear maximum principle of Pucci-Serrin [28] (p. 111). Hypothesis $\mathrm{H}_{0}$ (iv) serves the needs of our problem and it is mild. As we will see in the examples listed below, it is satisfied in all cases of interest.

Clearly the above hypotheses imply that the primitive $G_{0}(\cdot)$ is strictly convex and strictly increasing. Let $G(y)=G_{0}(|y|)$ for all $y \in \mathbb{R}^{N}$. Evidently $G \in C^{1}\left(\mathbb{R}^{N}, \mathbb{R}\right)$, it is convex and we have

$$
\begin{aligned}
& \nabla G(y)=G_{0}^{\prime}(|y|) \frac{y}{|y|}=a_{0}(|y|) y=a(y) \quad \text { for all } y \in \mathbb{R}^{N} \backslash\{0\}, \\
& \nabla G(0)=0 .
\end{aligned}
$$

So, $G(\cdot)$ is the primitive of $a(\cdot)$ and on account of the convexity of $G(\cdot)$, we have

$$
G(y) \leq(a(y), y)_{\mathbb{R}^{N}} \quad \text { for all } y \in \mathbb{R}^{N} .
$$

Hypotheses $\mathrm{H}_{0}$ lead easily to the following properties of the map $a(\cdot)$. 
Lemma 2 The mapping $a(\cdot)$ is continuous, strictly monotone (hence maximal monotone too) and

(a) $|a(y)| \leq c_{4}\left(|y|^{s-1}+|y|^{p-1}\right)$ for some $c_{4}>0$, all $y \in \mathbb{R}^{N}$;

(b) $\frac{c_{1}}{p-1}|y|^{p} \leq(a(y), y)_{\mathbb{R}^{N}}$ for all $y \in \mathbb{R}^{N}$.

Using this lemma and (1) we are led to the following growth restrictions on $G(\cdot)$.

Corollary 3 We have $\frac{c_{1}}{p-1}|y|^{p} \leq G(y) \leq c_{5}\left(1+|y|^{p}\right)$ for some $c_{5}>0$, all $y \in \mathbb{R}^{N}$.

Examples The following maps $a(\cdot)$ satisfy hypotheses $\mathrm{H}_{0}$ (see Papageorgiou-Rădulescu [19]):

(i) $a(y)=|y|^{p-2} y$ with $1<p<\infty$.

This map corresponds to the $p$-Laplace differential operator defined by

$$
\Delta_{p} u=\operatorname{div}\left(|D u|^{p-2} D u\right) \quad \text { for all } u \in W_{0}^{1, p}(\Omega) .
$$

(ii) $a(y)=|y|^{p-2} y+|y|^{q-2} y$ with $1<q<p$.

This map corresponds to the $(p, q)$-Laplace differential operator defined by

$$
\Delta_{p} u+\Delta_{q} u \quad \text { for all } u \in W_{0}^{1, p}(\Omega) .
$$

Such operators arise often in the mathematical models of physical processes and recently there have been published several works dealing with equations driven by such operators. We mention the works of Bobkov-Tanaka [3], Papageorgiou-Zhang [26, 27], Rădulescu [29], Ragusa-Tachikawa [30].

(iii) $a(y)=\left(1+|y|^{2}\right)^{\frac{p-2}{2}} y$ with $1<p<\infty$.

This map corresponds to the extended capillary differential operator defined by

$$
\operatorname{div}\left(\left(1+|D u|^{2}\right)^{\frac{p-2}{2}} D u\right) \quad \text { for all } u \in W_{0}^{1, p}(\Omega)
$$

Let $A: W_{0}^{1, p}(\Omega) \mapsto W^{-1, p^{\prime}}(\Omega)=W_{0}^{1, p}(\Omega)^{*}\left(\frac{1}{p}+\frac{1}{p^{\prime}}=1\right)$ be the nonlinear operator defined by

$$
\langle A(u), h\rangle=\int_{\Omega}(a(D u), D h)_{\mathbb{R}^{N}} d z \quad \text { for all } u, h \in W_{0}^{1, p}(\Omega)
$$

From Gasinski-Papageorgiou [9] (Problem 2.192, p. 279), we have the following properties for this operator.

Proposition 4 The operator $A(\cdot)$ is bounded (that is, maps bounded sets to bounded sets), continuous, strictly monotone (hence maximal monotone too) and of type $(S)_{+}$, that is,

$$
\begin{aligned}
& \text { “ } u_{n} \stackrel{w}{\rightarrow} u \quad \text { in } W_{0}^{1, p}(\Omega) \quad \text { and } \quad \limsup _{n \rightarrow \infty}\left|A\left(u_{n}\right), u_{n}-u\right\rangle \leq 0 \\
& \Rightarrow \quad u_{n} \rightarrow u \quad \text { in } W_{0}^{1, p}(\Omega) . "
\end{aligned}
$$


In the sequel by $\hat{\lambda}_{1}(r)$ we denote the first (principal) eigenvalue of $\left(-\Delta_{r}, W_{0}^{1, r}(\Omega)\right)(1<$ $r<\infty)$. We know that

- $\quad \hat{\lambda}_{1}(r)>0, \quad$ it is simple and isolated.

- $\quad \hat{\lambda}_{1}(r)=\inf \left\{\frac{\|D u\|_{r}^{r}}{\|u\|_{r}^{r}}: u \in W_{0}^{1, r}(\Omega), u \neq 0\right\}$.

In (2) the infimum is realized on the corresponding one-dimensional eigenspace the elements of which do not change sign. By $\hat{u}_{1}(r)$ we denote the $L^{r}$-normalized (that is, $\left.\left\|\hat{u}_{1}(r)\right\|_{r}=1\right)$, positive eigenfunction corresponding to $\hat{\lambda}_{1}(r)$. The nonlinear regularity theory and the nonlinear maximum principle (see Gasinski-Papageorgiou [8], pp. 738-739), imply $\hat{u}_{1}(r) \in \operatorname{int} C_{+}$. So, we have $\left\|D \hat{u}_{1}(r)\right\|_{r}^{r}=\hat{\lambda}_{1}(r)$. We mention that every eigenvalue $\hat{\lambda}$ of $\left(-\Delta_{r}, W_{0}^{1, r}(\Omega)\right)$ distinct from $\hat{\lambda}_{1}(r)$ has eigenfunctions which are nodal (sign-changing).

Given $x \in \mathbb{R}$, we set $x^{ \pm}=\max \{ \pm x, 0\}$. Then for $u \in W_{0}^{1, p}(\Omega)$ we define $u^{ \pm}(z)=u(z)^{ \pm}$for all $z \in \Omega$. We know that

$$
u^{ \pm} \in W_{0}^{1, p}(\Omega), \quad u=u^{+}-u^{-}, \quad|u|=u^{+}+u^{-} .
$$

If $u, v: \Omega \mapsto \mathbb{R}$ are measurable functions and $u \leq v$, then we define

$$
[u, v]=\left\{h \in W_{0}^{1, p}(\Omega): u(z) \leq h(z) \leq v(z) \text { for a.a. } z \in \Omega\right\} .
$$

A set $S \subseteq W_{0}^{1, p}(\Omega)$ is said to be the downward directed, if for every $u, v \in S$, we can find $y \in S$ such that $y \leq u$ and $y \leq v$.

Now we introduce the hypotheses on perturbation $f(z, x, y)$.

$\mathrm{H}_{1}: f: \Omega \times \mathbb{R} \times \mathbb{R}^{N} \mapsto \mathbb{R}$ is a Carathéodory function, $f(z, 0, y)=0$ for a.a. $z \in \Omega$, all $y \in \mathbb{R}^{N}$ and

(i) there exist $0<\delta_{0}<\theta$ and $c_{6}>0$ such that

$$
\begin{aligned}
& |f(z, x, y)| \leq c_{6}\left(1+|y|^{p-1}\right) \quad \text { for a.a. } z \in \Omega \text {, all } 0 \leq x \leq \theta \text {, all } y \in \mathbb{R}^{N}, \\
& \theta^{-\eta}+f(z, \theta, y) \leq 0, \quad \text { for a.a. } z \in \Omega \text {, all } y \in \mathbb{R}^{N}, \\
& f(z, x, y) \geq 0 \quad \text { for a.a. } z \in \Omega \text {, all } 0 \leq x \leq \delta_{0} \text {, all } y \in \mathbb{R}^{N} ;
\end{aligned}
$$

(ii) for every $M>0$, there exists $\eta_{M} \in L^{\infty}(\Omega)$ such that

$$
\begin{aligned}
& \eta_{M}(z) \geq \frac{c_{*}}{q} \hat{\lambda}_{1}(q) \quad \text { for a.a. } z \in \Omega, \quad \eta_{M} \not \equiv \frac{c_{*}}{q} \hat{\lambda}_{1}(q), \\
& \liminf _{x \rightarrow 0^{+}} \frac{f(z, x, y)}{x^{q-1}} \geq \eta_{M}(z) \quad \text { uniformly for a.a. } z \in \Omega, \text { all }|y| \leq M ;
\end{aligned}
$$

(iii) for a.a. $z \in \Omega$, all $0 \leq x \leq \theta$, all $y \in \mathbb{R}^{N}$ and all $0<t<1$, we have

$$
f\left(z, \frac{1}{t} x, y\right) \leq \frac{1}{t^{p-1}} f(z, x, y) .
$$

Remark 2 The above hypotheses concern only the behavior of $f(z, \cdot, y)$ near zero. No global growth condition is imposed on $f(z, \cdot, y)$. Hypothesis $\mathrm{H}_{1}(\mathrm{i})$ dictates an oscillatory behavior for $f(z, \cdot, y)$. It starts positive and by the time we have reached $x=\theta$, the perturbation 
$f(z, \cdot, y)$ has become negative. In the case of the equation driven by the $q$-Laplacian, hypothesis $\mathrm{H}_{1}$ (ii) is a nonuniform nonresonance condition at zero. Hypothesis $\mathrm{H}_{1}$ (iii) is satisfied if for a.a. $z \in \Omega$ and all $y \in \mathbb{R}^{N}$ the quotient function $x \mapsto \frac{f(z, x, y)}{x^{p-1}}$ is nonincreasing on $(0, \theta]$.

Example The following function satisfies hypotheses $\mathrm{H}_{1}$ above. For the sake of simplicity, we drop the $z$-dependence. We have

$$
f(x, y)=\eta\left(x^{p-1}-2 x^{r-1}\right)\left(1+|y|^{p-1}\right) \quad \text { for all } x \geq 0 \text {, all } y \in \mathbb{R}^{N},
$$

with $\eta>\max \left\{\frac{c_{*}}{q} \hat{\lambda}_{1}(q), 1\right\}, 1<q<r$. For $\theta=1$ hypothesis $\mathrm{H}_{1}(\mathrm{i})$ is satisfied.

As we already mentioned in the Introduction, due to the presence of the singular term, we have an energy functional which is not $C^{1}$ and this prevents us from using the tools of critical point theory. So, we need to find a way to bypass the singularity and deal with $C^{1}$ functionals. For this reason in the next section we deal with the purely singular problem $(f \equiv 0)$. A solution of this problem will help us isolate the singularity.

\section{Purely singular problem}

In this section we examine the following purely singular problem:

$$
-\operatorname{div} a(D u(z))=\lambda u(z)^{-\eta} \quad \text { in } \Omega,\left.u\right|_{\partial \Omega}=0, u>0 .
$$

Proposition 5 If hypotheses $\mathrm{H}_{0}$ hold, then for every $\lambda>0$ problem $\left(Q_{\lambda}\right)$ admits a unique solution $\bar{u} \in \operatorname{int} C_{+}$and $\bar{u}_{\lambda} \rightarrow 0$ in $C_{0}^{1}(\bar{\Omega})$ as $\lambda \rightarrow 0^{+}$.

Proof Let $g \in L^{p}(\Omega)$ and $\varepsilon>0$. We consider the following auxiliary Dirichlet problem:

$$
-\operatorname{div} a(D u(z))=\frac{\lambda}{(|g(z)|+\varepsilon)^{\eta}} \quad \text { in } \Omega,\left.u\right|_{\partial \Omega}=0, u \geq 0
$$

Recall that $A: W_{0}^{1, p}(\Omega) \mapsto W^{-1, p^{\prime}}(\Omega)$ is the operator defined by

$$
\left.\langle A(u), h\rangle=\int_{\Omega}(a(D u)), D h\right)_{\mathbb{R}^{N}} d z \quad \text { for all } u, h \in W_{0}^{1, p}(\Omega) .
$$

On account of Proposition 4, the operator $A(\cdot)$ is continuous and maximal monotone. In addition, from Lemma 2(b) we see that $A(\cdot)$ is coercive. Therefore $A(\cdot)$ is surjective (see Papageorgiou-Rădulescu-Repovš [21], Corollary 2.8.7, p. 135). So, we can find $\hat{u}_{\varepsilon} \in$ $W_{0}^{1, p}(\Omega)$ such that

$$
A\left(\hat{u}_{\varepsilon}\right)=\frac{\lambda}{(|g(z)|+\varepsilon)^{\eta}}
$$

Moreover, the strict monotonicity of $A(\cdot)$ implies that this solution is unique. Note that $\frac{\lambda}{(|g(\cdot)|+\varepsilon)^{\eta}} \in L^{\infty}(\Omega)_{+}$. So, the nonlinear regularity theory of Lieberman [17] and the nonlinear maximum principle of Pucci-Serrin [28] (p. 111,120) imply that $\hat{u}_{\varepsilon} \in \operatorname{int} C_{+}$. 
We consider the solution map $\gamma_{\varepsilon}: L^{p}(\Omega) \mapsto L^{p}(\Omega)$ defined by

$$
\gamma_{\varepsilon}(g)=\hat{u}_{\varepsilon}
$$

Consider the following perturbation of problem $\left(Q_{\lambda}\right)$ :

$$
-\operatorname{div} a(D u(z))=\frac{\lambda}{(u(z)+\varepsilon)^{\eta}} \quad \text { in } \Omega,\left.u\right|_{\partial \Omega}=0, u \geq 0 .
$$

Evidently a fixed point of $\gamma_{\varepsilon}(\cdot)$ is a solution of $\left(Q_{\lambda}^{\varepsilon}\right)$. Clearly $\gamma_{\varepsilon}(\cdot)$ is continuous. Moreover, using Lemma 2(b) we have

$$
\begin{aligned}
& \frac{c_{1}}{p-1}\left\|D \hat{u}_{\varepsilon}\right\|_{p}^{p} \leq \int_{\Omega} \frac{\lambda}{(|g(z)|+\varepsilon)^{\eta}} \hat{u}_{\varepsilon} d z \leq \frac{\lambda}{\varepsilon^{\eta}} c_{7}\left\|\hat{u}_{\varepsilon}\right\| \text { for some } c_{7}>0 \\
& \Rightarrow \quad \gamma_{\varepsilon}\left(L^{p}(\Omega)\right) \subseteq W_{0}^{1, p}(\Omega) \text { is bounded. }
\end{aligned}
$$

The fact that $W_{0}^{1, p}(\Omega) \hookrightarrow L^{p}(\Omega)$ compactly implies that

$$
\gamma_{\varepsilon}\left(L^{p}(\Omega)\right) \subseteq L^{p}(\Omega) \text { is relatively compact. }
$$

Then, by the Schauder-Tychonov fixed point theorem (see Papageorgiou-RădulescuRepovš [21], Theorem 4.3.21, p. 298), we can find $\widetilde{u}_{\varepsilon} \in W_{0}^{1, p}(\Omega)$ such that

$$
\gamma_{\varepsilon}\left(\widetilde{u}_{\varepsilon}\right)=\widetilde{u}_{\varepsilon}
$$

This is the unique positive solution of $\left(Q_{\lambda}^{\varepsilon}\right)$ and since $\gamma_{\varepsilon}(\cdot)$ has values in int $C_{+}$, we infer that $\widetilde{u}_{\varepsilon} \in \operatorname{int} C_{+}$.

Claim: $\left\{\widetilde{u}_{\varepsilon}\right\}_{\varepsilon \in(0,1]} \subseteq \operatorname{int} C_{+}$is nonincreasing.

Let $0<\varepsilon^{\prime}<\varepsilon$. We have

$$
-\operatorname{div} a\left(D \widetilde{u}_{\varepsilon^{\prime}}\right)=\frac{\lambda}{\left(\widetilde{u}_{\varepsilon^{\prime}}+\varepsilon^{\prime}\right)^{\eta}} \geq \frac{\lambda}{\left(\widetilde{u}_{\varepsilon^{\prime}}+\varepsilon\right)^{\eta}} \quad \text { in } \Omega
$$

We introduce the Carathéodory function $k_{\varepsilon}: \Omega \times \mathbb{R} \mapsto \mathbb{R}$ defined by

$$
k_{\varepsilon}(z, x)= \begin{cases}\frac{\lambda}{\left(x^{+}+\varepsilon\right)^{\eta}} & \text { if } x \leq \widetilde{u}_{\varepsilon^{\prime}}(z), \\ \frac{\lambda}{\left(\widetilde{u}_{\varepsilon^{\prime}}(z)+\varepsilon\right)^{\eta}} & \text { if } \widetilde{u}_{\varepsilon^{\prime}}(z)<x .\end{cases}
$$

We set $K_{\varepsilon}(z, x)=\int_{0}^{x} k_{\varepsilon}(z, s) d s$ and consider the $C^{1}$-functional $\psi_{\varepsilon}: W_{0}^{1, p}(\Omega) \mapsto \mathbb{R}$ defined by

$$
\psi_{\varepsilon}(u)=\int_{\Omega} G(D u) d z-\int_{\Omega} K_{\varepsilon}(z, u) d z \quad \text { for all } u \in W_{0}^{1, p}(\Omega) .
$$

From Corollary 3 and (4), we see that the functional $\psi_{\varepsilon}(\cdot)$ is coercive. Also, using the Sobolev embedding theorem, we see that $\psi_{\varepsilon}(\cdot)$ is sequentially weakly lower semicontinuous. So, by the Weierstrass-Tonelli theorem, we can find $\widetilde{u}_{\varepsilon}^{*} \in W_{0}^{1, p}(\Omega)$ such that

$$
\psi_{\varepsilon}\left(\widetilde{u}_{\varepsilon}^{*}\right)=\min \left\{\psi_{\varepsilon}(u): u \in W_{0}^{1, p}(\Omega)\right\} .
$$


From hypothesis $\mathrm{H}_{0}$ (iv) and Corollary 3, we have

$$
G(y) \leq c_{8}\left(|y|^{q}+|y|^{p}\right) \quad \text { for some } c_{8}>0 \text {, all } y \in \mathbb{R}^{N} \text {. }
$$

For $t \in(0,1)$ we have

$$
\psi_{\varepsilon}\left(t \tilde{u}_{\varepsilon^{\prime}}\right) \leq \frac{t^{p}}{p}\left\|D \widetilde{u}_{\varepsilon^{\prime}}\right\|_{p}^{p}+\frac{t^{q}}{q}\left\|D \widetilde{u}_{\varepsilon^{\prime}}\right\|_{q}^{q}-\frac{t^{1-\eta}}{1-\eta} \int_{\Omega} \tilde{u}_{\varepsilon^{\prime}}^{1-\eta} d z \quad \text { (see (6)). }
$$

Since $0<\eta<1<q<p$, for $t \in(0,1)$ small, we see that

$$
\begin{aligned}
& \psi_{\varepsilon}\left(t \tilde{u}_{\varepsilon^{\prime}}\right)<0 \\
& \Rightarrow \quad \psi_{\varepsilon}\left(\tilde{u}_{\varepsilon}^{*}\right)<0=\psi_{\varepsilon}(0) \quad(\operatorname{see}(5)) \\
& \Rightarrow \quad \tilde{u}_{\varepsilon}^{*} \neq 0 .
\end{aligned}
$$

From (5) we have

$$
\begin{aligned}
& \psi_{\varepsilon}\left(\widetilde{u}_{\varepsilon}^{*}\right)=0 \\
& \Rightarrow \quad\left\langle A\left(\widetilde{u}_{\varepsilon}^{*}\right), h\right\rangle=\int_{\Omega} k_{\varepsilon}\left(z, \tilde{u}_{\varepsilon}^{*}\right) h d z \quad \text { for all } h \in W_{0}^{1, p}(\Omega) .
\end{aligned}
$$

In (7) first we choose $h=-\left(\widetilde{u}_{\varepsilon}^{*}\right)^{-} \in W_{0}^{1, p}(\Omega)$. We have

$$
\begin{aligned}
& \frac{c_{1}}{p-1}\left\|D\left(\widetilde{u}_{\varepsilon}^{*}\right)^{-}\right\|_{p}^{p} \leq 0 \quad(\text { see Lemma } 2(\mathrm{~b}) \text { and }(4)) \\
& \Rightarrow \quad \tilde{u}_{\varepsilon}^{*} \geq 0, \quad \tilde{u}_{\varepsilon}^{*} \neq 0 .
\end{aligned}
$$

Next we test (7) with $h=\left(\widetilde{u}_{\varepsilon}^{*}-\widetilde{u}_{\mathcal{E}^{\prime}}\right)^{+} \in W_{0}^{1, p}(\Omega)$. We have

$$
\begin{aligned}
\left\langle A\left(\widetilde{u}_{\varepsilon}^{*}\right),\left(\widetilde{u}_{\varepsilon}^{*}-\tilde{u}_{\varepsilon^{\prime}}\right)^{+}\right\rangle & =\int_{\Omega} \frac{\lambda}{\left(\widetilde{u}_{\varepsilon^{\prime}}+\varepsilon\right)^{\eta}}\left(\widetilde{u}_{\varepsilon}^{*}-\widetilde{u}_{\varepsilon^{\prime}}\right)^{+} d z \quad(\text { see }(4)) \\
& \leq\left\langle A\left(\widetilde{u}_{\varepsilon^{\prime}}\right),\left(\tilde{u}_{\varepsilon}^{*}-\widetilde{u}_{\varepsilon^{\prime}}\right)^{+}\right\rangle \quad(\text { see }(3)) \\
\Rightarrow \quad \tilde{u}_{\varepsilon}^{*} \leq \tilde{u}_{\varepsilon^{\prime}} . &
\end{aligned}
$$

So, we have proved that

$$
\tilde{u}_{\varepsilon}^{*} \in\left[0, \tilde{u}_{\varepsilon^{\prime}}\right], \quad \tilde{u}_{\varepsilon}^{*} \neq 0 .
$$

From (8), (4) and (7) it follows that

$$
\begin{aligned}
\tilde{u}_{\varepsilon}^{*} & =\tilde{u}_{\varepsilon} \\
& \Rightarrow \quad \tilde{u}_{\varepsilon} \leq \tilde{u}_{\varepsilon^{\prime}} .
\end{aligned}
$$

This proves the claim. 
Next, let $\varepsilon_{n}=\frac{1}{n}$ and $\widetilde{u}_{n}=\widetilde{u}_{\varepsilon_{n}} \in \operatorname{int} C_{+}$for all $n \in \mathbb{N}$. We have

$$
\begin{aligned}
& \left\langle A\left(\widetilde{u}_{n}\right), h\right\rangle=\int_{\Omega} \frac{\lambda}{\left(\widetilde{u}_{n}+\frac{1}{n}\right)^{\eta}} h d z \text { for all } h \in W_{0}^{1, p}(\Omega) \text {, all } n \in \mathbb{N}, \\
& \tilde{u}_{1} \leq \tilde{u}_{n} \text { for all } n \in \mathbb{N} \text { (by the claim). }
\end{aligned}
$$

Consider the Banach space $C_{0}(\bar{\Omega})=\left\{u \in C(\bar{\Omega}):\left.u\right|_{\partial \Omega}=0\right\}$ (with the supremum norm). This is an ordered Banach space with positive cone

$$
K_{+}=\left\{u \in C_{0}(\bar{\Omega}): u(z) \geq 0 \text { for all } z \in \bar{\Omega}\right\}
$$

This cone has a nonempty interior given by

$$
\operatorname{int} K_{+}=\left\{u \in C_{+}: c_{u} \hat{d} \leq u \text { for some } c_{u}>0\right\} \text {, }
$$

with $\hat{d}(z)=d(z, \partial \Omega)$ for all $z \in \bar{\Omega}$. Since $\widetilde{u}_{1} \in \operatorname{int} C_{+}$, we can find $0<c_{9}<c_{10}$ such that

$$
c_{9} \hat{d} \leq \widetilde{u}_{1} \leq c_{10} \hat{d} \quad \text { (see Guo-Webb [13], Lemma 2.3). }
$$

Let $s>N$ and note that $\hat{u}_{1}(p)^{1 / s} \in K_{+}$. Using Proposition 4.1.22, p. 274, of PapageorgiouRădulescu-Repovš [21], we can find $c_{11}>0$ such that

$$
\begin{aligned}
& \hat{u}_{1}(p)^{1 / s} \leq c_{11} \hat{d} \\
& \quad \Rightarrow \quad \hat{d}^{-\eta} \leq c_{12} \hat{u}_{1}(p)^{-\eta / s} \text { for some } c_{12}>0 .
\end{aligned}
$$

Since $0<\eta<1$, the Lemma in Lazer-McKenna [16] implies that $\hat{u}_{1}(p)^{-\eta / s} \in L^{s}(\Omega)$. Therefore

$$
\hat{d}^{-\eta} \in L^{s}(\Omega) \quad(\text { see }(12))
$$

From (11) we have

$$
\begin{aligned}
\tilde{u}_{1}^{-\eta} & \leq c_{13} \hat{d}^{-\eta} \quad \text { for some } c_{13}>0 \\
\Rightarrow & \tilde{u}_{1}^{-\eta} \in L^{s}(\Omega) \quad(\text { see }(13)) .
\end{aligned}
$$

We return to (9) and use $h=\widetilde{u}_{n} \in W_{0}^{1, p}(\Omega)$. Then from Lemma 2(b) we have

$$
\begin{aligned}
& \frac{c_{1}}{p-1}\left\|D \widetilde{u}_{n}\right\|_{p}^{p} \leq \int_{\Omega} \frac{\lambda}{\left(\widetilde{u}_{n}+\frac{1}{n}\right)^{\eta}} \widetilde{u}_{n} d z \leq \lambda c_{14}\left\|\widetilde{u}_{n}\right\|^{1-\eta} \quad \text { for some } c_{14}>0 \text {, all } n \in \mathbb{N} \\
& \Rightarrow \quad\left\{\widetilde{u}_{n}\right\}_{n \in \mathbb{N}} \subseteq W_{0}^{1, p}(\Omega) \text { is bounded. }
\end{aligned}
$$

So, we may assume that

$$
\tilde{u}_{n} \stackrel{w}{\rightarrow} \bar{u}_{\lambda} \quad \text { in } W_{0}^{1, p}(\Omega) \quad \text { and } \quad \tilde{u}_{n} \rightarrow \bar{u}_{\lambda} \quad \text { in } L^{p}(\Omega)
$$


Note that

$$
0 \leq \frac{\lambda}{\left(\widetilde{u}_{n}+\frac{1}{n}\right)^{\eta}} \leq \frac{\lambda}{\widetilde{u}_{1}^{\eta}} \in L^{s}(\Omega) \quad(\text { see }(14)) .
$$

On account of (15), we can also say (at least for a subsequence) that

$$
\frac{\lambda}{\left(\widetilde{u}_{n}(z)+\frac{1}{n}\right)^{\eta}} \rightarrow \frac{\lambda}{\bar{u}_{\lambda}(z)^{\eta}} \quad \text { for a.a. } z \in \Omega \text {. }
$$

From (16) and (17) it follows that

$$
\frac{\lambda}{\left(\widetilde{u}_{n}+\frac{1}{n}\right)^{\eta}} \stackrel{w}{\rightarrow} \frac{\lambda}{\bar{u}_{\lambda}{ }^{\eta}} \quad \text { in } L^{s}(\Omega)
$$

(see Gasinski-Papageorgiou [9], Problem 1.19, p. 38).

Again we return to (9) and choose $h=\widetilde{u}_{n}-\bar{u}_{\lambda} \in W_{0}^{1, p}(\Omega)$, pass to the limit as $n \rightarrow \infty$ and use (15) and (18). We obtain

$$
\begin{aligned}
& \lim _{n \rightarrow \infty}\left\langle A\left(\widetilde{u}_{n}\right), \widetilde{u}_{n}-\bar{u}_{\lambda}\right\rangle=0 \\
& \quad \Rightarrow \quad \widetilde{u}_{n} \rightarrow \bar{u}_{\lambda} \text { in } W_{0}^{1, p}(\Omega) \quad \text { (see Proposition 4). }
\end{aligned}
$$

Therefore if in (9) we pass to the limit as $n \rightarrow \infty$ and use (19), then

$$
\left\{\begin{array}{ll}
\left\langle A\left(\bar{u}_{\lambda}\right), h\right\rangle=\int_{\Omega} \frac{\lambda}{\bar{u}_{\lambda} \eta} h d z \quad \text { for all } h \in W_{0}^{1, p}(\Omega), \\
\widetilde{u}_{1} \leq \bar{u}_{\lambda} & (\text { see }(10)) .
\end{array}\right\}
$$

From (20) we infer that

$\bar{u}_{\lambda}$ is a positive solution of $\left(Q_{\lambda}\right)$.

Let $\theta=\frac{\lambda}{\overline{u_{\lambda} \eta}} \in L^{s}(\Omega)$ and consider the linear Dirichlet problem

$$
-\Delta y(z)=\theta(z) \quad \text { in } \Omega,\left.y\right|_{\partial \Omega}=0 .
$$

From Theorem 9.15, p. 241, of Gilbarg-Trudinger [12], we know that this problem has a unique solution $y \in W^{2, s}(\Omega), y \geq 0, y \neq 0$. Since $s>N$, by the Sobolev embedding theorem, we have $W^{2, s}(\Omega) \hookrightarrow C^{1, \alpha}(\bar{\Omega})$ continuously with $\alpha=1-\frac{N}{s} \in(0,1)$. Let $w=D y \in C^{0, \alpha}\left(\bar{\Omega}, \mathbb{R}^{N}\right)$. We have

$$
-\operatorname{div}\left(a\left(D \bar{u}_{\lambda}(z)\right)-w(z)\right)=0, \quad \text { in } \Omega,\left.\bar{u}_{\lambda}\right|_{\partial \Omega}=0 .
$$

Then the nonlinear regularity theory of Lieberman [17] implies that $\bar{u}_{\lambda} \in C_{+} \backslash\{0\}$. Using the nonlinear maximum principle of Pucci-Serrin [28] (pp. 111, 120), we infer that $\bar{u}_{\lambda} \in$ int $C_{+}$.

From (20) and (14) and Theorem 7.1, p. 286, of Ladyzhenskaya-Uraltseva [15] (see also Gasinski-Papageorgiou [8], p. 737), we know that there exists $c_{15}>0$ such that

$$
\left\|\bar{u}_{\lambda}\right\|_{\infty} \leq c_{15} \text { for all } 0<\lambda \leq 1
$$


Then the nonlinear regularity theory of Lieberman [17], says that we can find $\alpha \in(0,1)$ and $c_{16}>0$ such that

$$
\bar{u}_{\lambda} \in C_{0}^{1, \alpha}(\bar{\Omega})=C^{1, \alpha}(\bar{\Omega}) \cap C_{0}^{1}(\bar{\Omega}), \quad\left\|\bar{u}_{\lambda}\right\|_{C_{0}^{1, \alpha}(\bar{\Omega})} \leq c_{16} \quad \text { for all } \lambda \in(0,1]
$$

Since $C_{0}^{1, \alpha}(\bar{\Omega}) \hookrightarrow C_{0}^{1}(\bar{\Omega})$ compactly, from (14), (20) and (21), we conclude that

$$
\bar{u}_{\lambda} \rightarrow 0 \quad \text { in } C_{0}^{1}(\bar{\Omega}) \text { as } \lambda \rightarrow 0^{+} .
$$

This proof is now complete.

\section{The frozen variable method}

In this section we develop the method described in the Introduction (the frozen variable method).

So, fix $v \in C_{0}^{1}(\bar{\Omega})$ and consider the following Dirichlet problem (the "frozen problem"):

$$
\left\{\begin{array}{l}
-\operatorname{div} a(D u(z))=\lambda u(z)^{-\eta}+f(z, u(z), D v(z)) \quad \text { in } \Omega, \\
\left.u\right|_{\partial \Omega}=0, \quad u \geq 0, \quad \lambda>0, \quad 0<\eta<1 .
\end{array}\right\}
$$

Since we have fixed the gradient variable in $f$, the resulting problem $\left(P_{\lambda}^{v}\right)$ has a variational structure. However, as we already mentioned in the Introduction, the presence of the singular term $u \mapsto \lambda u^{-\eta}$ leads to an energy functional which is not $C^{1}$ and so we cannot use the minimax theorems of the critical point theory. To remedy this situation, we use the solution $\bar{u}_{\lambda}$ of $\left(Q_{\lambda}\right)$ to bypass the singularity and deal with a $C^{1}$-functional.

Let $M \geq\|D v\|_{\infty}$. On account of hypotheses $\mathrm{H}_{1}$ (ii), given $\varepsilon>0$ small, we can find $\delta \in$ $\left(0, \delta_{0}\right]$ such that

$$
f(z, x, y) \geq\left(\eta_{M}(z)-\varepsilon\right) x^{q-1} \quad \text { for a.a } z \in \Omega \text {, all } 0 \leq x \leq \delta \text {, all }|y| \leq M .
$$

Proposition 6 If hypotheses $\mathrm{H}_{0}, \mathrm{H}_{1}$ hold, then we can find $\hat{\lambda}_{*}>0$ such that for all $\lambda \in\left(0, \hat{\lambda}_{*}\right]$ problem $\left(P_{\lambda}^{v}\right)$ admits a positive solution $\hat{u}_{v} \in \operatorname{int} C_{+}$.

Proof Proposition 5 implies that we can find $\hat{\lambda}_{*} \in(0,1]$ such that

$$
0 \leq \bar{u}_{\lambda}(z) \leq \delta \quad \text { for all } z \in \bar{\Omega} \text {, all } 0<\lambda \leq \hat{\lambda}_{*}
$$

We introduce the Carathéodory function $\tau_{v}^{\lambda}: \Omega \times \mathbb{R} \mapsto \mathbb{R}$ defined by

$$
\tau_{v}^{\lambda}(z, x)= \begin{cases}\lambda \bar{u}_{\lambda}(z)^{-\eta}+f\left(z, \bar{u}_{\lambda}(z), D v(z)\right) & \text { if } x<\bar{u}_{\lambda}(z), \\ \lambda x^{-\eta}+f(z, x, D v(z)) & \text { if } \bar{u}_{\lambda}(z) \leq x \leq \theta, \\ \lambda \theta^{-\eta}+f(z, \theta, D v(z)) & \text { if } \theta \leq x\end{cases}
$$

recall that $\delta_{0}<\theta$, see hypothesis $\mathrm{H}_{1}(\mathrm{i})$. We set $T_{v}^{\lambda}(z, x)=\int_{0}^{x} \tau_{v}^{\lambda}(z, s) d s$ and consider the $C^{1}$-functional $\varphi_{v}^{\lambda}: W_{0}^{1, p}(\Omega) \mapsto \mathbb{R}$ defined by

$$
\varphi_{\nu}^{\lambda}(u)=\int_{\Omega} G(D u) d z-\int_{\Omega} T_{\nu}^{\lambda}(z, u) d z \quad \text { for all } u \in W_{0}^{1, p}(\Omega) .
$$


Corollary 3 and (24) imply that $\varphi_{v}^{\lambda}(\cdot)$ is coercive. Also, it is sequentially weakly lower semicontinuous. So, we can find $\hat{u}_{v} \in W_{0}^{1, p}(\Omega)$ such that

$$
\varphi_{v}^{\lambda}\left(\hat{u}_{v}\right)=\min \left\{\varphi_{v}^{\lambda}(u): u \in W_{0}^{1, p}(\Omega)\right\}
$$

Hypothesis $\mathrm{H}_{0}(\mathrm{iv})$ implies that, given $\varepsilon^{\prime} \in(0, \varepsilon]$, we can find $\delta^{\prime} \in(0, \delta]$ such that

$$
G(y) \leq \frac{c_{*}+\varepsilon^{\prime}}{q}|y|^{q} \quad \text { for all }|y| \leq \delta^{\prime} .
$$

Let $t \in(0,1)$ be small such that

$$
0 \leq t \hat{u}_{1}(q)(z) \leq \min \left\{\bar{u}_{\lambda}(z), \delta_{0}\right\} \quad \text { for all } z \in \bar{\Omega}
$$

(recall that $\bar{u}_{\lambda} \in \operatorname{int} C_{+}$and use Proposition 4.1.22, p. 274, of Papageorgiou-RădulescuRepovš [21]). We have

$$
\begin{aligned}
\varphi_{v}^{\lambda}\left(t \hat{u}_{1}(q)\right) \leq & \frac{t^{p}}{p}\left\|D \hat{u}_{1}(q)\right\|_{p}^{p}+\frac{t^{q}}{q}\left(c_{*}+\varepsilon^{\prime}\right) \hat{\lambda}_{1}(q)-\frac{t^{q}}{q} \int_{\Omega}\left(\eta_{M}(z)-\varepsilon\right) \hat{u}_{1}(q)^{q} d z \\
& (\text { see }(26),(22),(23),(24)) \\
\leq & \frac{t^{p}}{p}\left\|D \hat{u}_{1}(q)\right\|_{p}^{p}+\frac{t^{q}}{q}\left(\int_{\Omega}\left(c_{*} \hat{\lambda}_{1}(q)-\eta_{M}(z)\right) \hat{u}_{1}(q)^{q} d z+2 \varepsilon\right) \\
& \left(\text { recall }\left\|\hat{u}_{1}(q)\right\|_{q}=1\right) .
\end{aligned}
$$

Note that

$$
\mu=\int_{\Omega}\left(\eta_{M}(z)-c_{*} \hat{\lambda}_{1}(q)\right) \hat{u}_{1}^{q} d z>0
$$

(see hypothesis $\mathrm{H}_{1}(\mathrm{ii})$ and recall $\hat{u}_{1}(q) \in \operatorname{int} C_{+}$).

We have

$$
\varphi_{v}^{\lambda}\left(t \hat{u}_{1}(q)\right) \leq c_{17} t^{p}-c_{18} t^{q} \quad \text { for some } c_{17}, c_{18}>0 \text { (with } \varepsilon>0 \text { small). }
$$

Since $q<p$, choosing $t \in(0,1)$ even smaller, we have

$$
\begin{aligned}
& \varphi_{v}^{\lambda}\left(t \hat{u}_{1}(q)\right)<0 \\
& \quad \Rightarrow \quad \varphi_{v}^{\lambda}\left(\hat{u}_{v}\right)<0=\varphi_{v}^{\lambda}(0) \quad(\text { see }(25)) \\
& \quad \Rightarrow \quad \hat{u}_{v} \neq 0 .
\end{aligned}
$$

From (25) we have

$$
\begin{aligned}
& \left(\varphi_{v}^{\lambda}\right)^{\prime}\left(\hat{u}_{v}\right)=0 \\
& \quad \Rightarrow \quad\left\langle A\left(\hat{u}_{v}\right), h\right\rangle=\int_{\Omega} \tau_{v}^{\lambda}\left(z, \hat{u}_{v}\right) h d z \quad \text { for all } h \in W_{0}^{1, p}(\Omega) .
\end{aligned}
$$


In (27) first we choose $h=\left(\overline{\mathcal{u}}_{\lambda}-\hat{\mathcal{u}}_{v}\right)^{+} \in W_{0}^{1, p}(\Omega)$. Then

$$
\begin{aligned}
&\left\langle A\left(\hat{u}_{v}\right),\left(\bar{u}_{\lambda}-\hat{u}_{v}\right)^{+}\right\rangle \\
&=\int_{\Omega}\left(\lambda \bar{u}_{\lambda}^{-\eta}+f\left(z, \bar{u}_{\lambda}, D v\right)\right)\left(\bar{u}_{\lambda}-\hat{u}_{v}\right)^{+} d z \quad(\text { see }(24)) \\
& \geq \int_{\Omega} \lambda \bar{u}_{\lambda}^{-\eta}\left(\bar{u}_{\lambda}-\hat{u}_{v}\right)^{+} d z \\
&\left(\text { since } f\left(z, \bar{u}_{\lambda}, D v\right) \geq 0 \quad(\text { see }(23)) \text { and hypothesis } \mathrm{H}_{1}(\mathrm{ii})\right) \\
&=\left\langle A\left(\bar{u}_{\lambda}\right),\left(\bar{u}_{\lambda}-\hat{u}_{v}\right)^{+}\right\rangle \quad(\text { see Proposition } 5) \\
& \Rightarrow \bar{u}_{\lambda} \leq \hat{u}_{v} .
\end{aligned}
$$

Next, in (27) we choose $h=\left(\hat{u}_{v}-\theta\right)^{+} \in W_{0}^{1, p}(\Omega)$. Then

$$
\begin{aligned}
& \left\langle A\left(\hat{u}_{v}\right),\left(\hat{u}_{v}-\theta\right)^{+}\right\rangle \\
& \quad=\int_{\Omega}\left(\lambda \theta^{-\eta}+f(z, \theta, D v)\right)\left(\hat{u}_{v}-\theta\right)^{+} d z \quad(\text { see }(24)) \\
& \leq \int_{\Omega}\left(\theta^{-\eta}+f(z, \theta, D v)\right)\left(\hat{u}_{v}-\theta\right)^{+} d z \quad\left(\text { recall } 0<\lambda \leq \hat{\lambda}_{*} \leq 1\right) \\
& \quad \leq 0 \quad\left(\text { see hypothesis } \mathrm{H}_{1}(\mathrm{i})\right) \\
& \quad \Rightarrow \quad \hat{u}_{v} \leq \theta
\end{aligned}
$$

(by the weak comparison principle; see Pucci-Serrin [28], Theorem 3.4.1, p. 61).

So, we have proved that

$$
\hat{u}_{v} \in\left[\bar{u}_{\lambda}, \theta\right]
$$

From (28), (24) and (27), we infer that $\hat{u}_{v}$ is a positive solution of $\left(P_{\lambda}^{v}\right)$. Since $\bar{u}_{\lambda}^{-\eta} \in L^{s}(\Omega)$ $(s>N)$ (see (20) and (14)), reasoning as in the proof of Proposition 5 (see the part of the proof after (20)), we infer that $\hat{u}_{v} \in \operatorname{int} C_{+}$.

This proof is now complete.

Let $S_{v}^{\lambda}$ be the set of positive solutions of the "frozen problem" $\left(P_{\lambda}^{v}\right)$. We have just proved that for $v \in C_{0}^{1}(\bar{\Omega})$ and $0<\lambda \leq \hat{\lambda}_{*}$ we have

$$
\emptyset \neq S_{v}^{\lambda} \subseteq \operatorname{int} C_{+} .
$$

We will show that each of these solution sets has a smallest element. In this way we have a canonical procedure to choose an element from $S_{v}^{\lambda}$ as $v \in C_{0}^{1}(\bar{\Omega})$ varies. So, we define the minimal solution map on which we will use Theorem 1 (the Leray-Schauder alternative principle).

To produce a minimal element for the solution set $S_{v}^{\lambda}$, we need the following result providing a lower bound for the set $S_{v}^{\lambda}$.

Proposition 7 If hypotheses $\mathrm{H}_{0}, \mathrm{H}_{1}$ hold and $0<\lambda \leq \hat{\lambda}_{*}$, then $\bar{u}_{\lambda} \leq u$ for all $u \in S_{\nu}^{\lambda}$. 
Proof Let $u \in S_{v}^{\lambda}$ and consider the Carathéodory function $\hat{\mu}_{\lambda}(z, x)$ defined on $\Omega \times \stackrel{\circ}{\mathbb{R}}_{+}=$ $\Omega \times(0, \infty)$ by

$$
\hat{\mu}_{\lambda}(z, x)= \begin{cases}\lambda x^{-\eta} & \text { if } 0<x \leq u(z) \\ \lambda u(z)^{-\eta} & \text { if } u(z)<x\end{cases}
$$

We consider the following singular Dirichlet problem

$$
-\operatorname{div} a(D u)=\hat{\mu}_{\lambda}(z, u) \quad \text { in } \Omega,\left.u\right|_{\partial \Omega}=0, u \geq 0 .
$$

Reasoning as in the proof of Proposition 5, we show that problem (30) has a solution $\widetilde{u}_{\lambda} \in \operatorname{int} C_{+}$and using (29), we show that

$$
\tilde{u}_{\lambda} \in[0, u] .
$$

But then (29) and Proposition 5 imply that

$$
\begin{aligned}
\tilde{u}_{\lambda} & =\bar{u}_{\lambda} \\
& \Rightarrow \quad \bar{u}_{\lambda} \leq u \quad \text { for all } u \in S_{v}^{\lambda} .
\end{aligned}
$$

This proof is now complete.

Using this lower bound, we show the existence of a minimal element for the set $S_{v}^{\lambda}$.

Proposition 8 If hypotheses $\mathrm{H}_{0}, \mathrm{H}_{1}$ hold and $0<\lambda \leq \hat{\lambda}_{*}$, then problem $\left(P_{\lambda}^{v}\right)$ has a smallest positive solution $u_{v}^{*} \in \operatorname{int} C_{+}$such that

$$
0 \leq u_{v}^{*}(z) \leq \theta \quad \text { for all } z \in \bar{\Omega}
$$

Proof From Proposition 18 of Papageorgiou-Rădulescu-Repovš [23], we know that $S_{v}^{\lambda}$ is downward directed. Then using Lemma 3.10, p. 178, of Hu-Papageorgiou [14], we can find $\left\{u_{n}\right\}_{n \in \mathbb{N}} \subseteq S_{v}^{\lambda}$ decreasing such that

$$
\inf _{n \in \mathbb{N}} u_{n}=\inf S_{v}^{\lambda}
$$

From the proof of Proposition 6 we know that $S_{v}^{\lambda} \cap[0, \theta] \neq \emptyset$. So, without any loss of generality, we may assume that $\left\{u_{n}\right\}_{n \in \mathbb{N}} \subseteq[0, \theta]$. We have

$$
\begin{aligned}
& \left\langle A\left(u_{n}\right), h\right\rangle=\int_{\Omega}\left(\lambda u_{n}{ }^{-\eta}+f\left(z, u_{n}, D v\right)\right) h d z \quad \text { for all } h \in W_{0}^{1, p}(\Omega), \text { all } n \in \mathbb{N}, \\
& \bar{u}_{\lambda} \leq u_{n} \leq u_{1} \quad \text { for all } n \in \mathbb{N} \text { (see Proposition 7). }
\end{aligned}
$$

In (31) we choose $h=u_{n} \in W_{0}^{1, p}(\Omega)$. Using Lemma 2(b), (32) and hypothesis $\mathrm{H}_{1}(\mathrm{i})$, we infer that

$$
\left\{u_{n}\right\}_{n \in \mathbb{N}} \subseteq W_{0}^{1, p}(\Omega) \text { is bounded. }
$$


So, we may assume that

$$
u_{n} \stackrel{w}{\rightarrow} u_{v}^{*} \quad \text { in } W_{0}^{1, p}(\Omega) \quad \text { and } \quad u_{n} \rightarrow u_{v}^{*} \quad \text { in } L^{p}(\Omega)
$$

In (31) we choose $h=u_{n}-u_{v}^{*} \in W_{0}^{1, p}(\Omega)$, pass to limit as $n \rightarrow \infty$ and use (33). We obtain

$$
\begin{gathered}
\lim _{n \rightarrow \infty}\left\langle A\left(u_{n}\right), u_{n}-u_{v}^{*}\right\rangle=0 \quad(\text { see the proof Proposition 5) } \\
\left.\Rightarrow \quad u_{n} \rightarrow u_{v}^{*} \quad \text { in } W_{0}^{1, p}(\Omega) \text { (see Proposition } 4\right)
\end{gathered}
$$

Then, if in (31) we pass to the limit as $n \rightarrow \infty$ and use (34), we obtain

$$
\left\langle A\left(u_{v}^{*}\right), h\right\rangle=\int_{\Omega}\left(\lambda\left(u_{v}^{*}\right)^{-\eta}+f\left(z, u_{v}^{*}, D v\right)\right) h d z \quad \text { for all } h \in W_{0}^{1, p}(\Omega) \text { and } \bar{u}_{\lambda} \leq u_{v}^{*} \text {. }
$$

It follows that

$$
u_{v}^{*} \in S_{v}^{\lambda}, \quad u_{v}^{*}=\inf S_{v}^{\lambda}, \quad 0 \leq u_{v}^{*}(z) \leq \theta \quad \text { for all } z \in \bar{\Omega} .
$$

This proof is now complete.

Using Proposition 8, we define the minimal solution map

$$
\xi_{\lambda}: C_{0}^{1}(\bar{\Omega}) \mapsto C_{0}^{1}(\bar{\Omega}), \quad \lambda \in\left(0, \hat{\lambda}_{*}\right]
$$

by setting

$$
\xi_{\lambda}(v)=u_{v}^{*} \quad \text { for all } v \in C_{0}^{1}(\bar{\Omega})
$$

Evidently a fixed point of $\xi_{\lambda}(\cdot)$ is a solution of problem $\left(P_{\lambda}\right)$. To produce a fixed point of $\left(P_{\lambda}\right)$, we will use Theorem 1 (the Leray-Schauder alternative principle). This is done in the next section.

\section{Positive solution}

To apply Theorem 1 , we need to know that $\xi_{\lambda}(\cdot)$ is compact. The next proposition will be helpful in this respect.

Proposition 9 If hypotheses $\mathrm{H}_{0}, \mathrm{H}_{1}$ hold, $v_{n} \rightarrow v$ in $C_{0}^{1}(\bar{\Omega}), u \in S_{v}^{\lambda} \cap[0, \theta]$ with $\lambda \in\left(0, \hat{\lambda}_{*}\right]$, then we can find $u_{n} \in S_{v_{n}}^{\lambda}, n \in \mathbb{N}$ such that

$$
u_{n} \rightarrow u \quad \text { in } C_{0}^{1}(\bar{\Omega})
$$

Proof Choosing $M \geq \sup _{n \in \mathbb{N}}\left\|D v_{n}\right\|_{\infty}$, we see that all the previous results are valid. We consider the following Dirichlet problem:

$$
-\operatorname{div} a(D y(z))=\tau_{v_{n}}^{\lambda}(z, u(z)) \quad \text { in } \Omega,\left.y\right|_{\partial \Omega}=0(\text { see }(24))
$$


This problem has a unique solution $y_{n} \in W_{0}^{1, p}(\Omega)$. We know that $\bar{u}_{\lambda}{ }^{-\eta} \in L^{s}(\Omega), s>N$ (see (20) and (14)). Therefore $\left\{\tau_{v_{n}}^{\lambda}(\cdot, u(\cdot))\right\}_{n \in \mathbb{N}} \subseteq L^{s}(\Omega)$ is bounded (see (24)). Consider the linear Dirichlet problem

$$
-\Delta e(z)=\tau_{v_{n}}^{\lambda}(z, u(z)) \quad \text { in } \Omega,\left.e\right|_{\partial \Omega}=0 .
$$

For each $n \in \mathbb{N}$, this problem has a unique solution $e_{n} \in W^{2, s}(\Omega)$ (see Gilbarg-Trudinger [12], Theorem 9.15, p. 241) and in addition we have

$$
\left\|e_{n}\right\|_{W^{2, s}(\Omega)} \leq c_{19} \quad \text { for some } c_{19}>0 \text {, all } n \in \mathbb{N}
$$

(see Gilbarg-Trudinger [12], Lemma 9.17, p. 242). From the Sobolev embedding theorem we know that, for $\alpha=1-\frac{N}{s} \in(0,1)$, we have

$$
W^{2, s}(\Omega) \hookrightarrow C^{1, \alpha}(\bar{\Omega}) \text { compactly. }
$$

Hence from (36) it follows that

$$
\left\{\hat{\gamma}_{n}=D e_{n}\right\}_{n \in \mathbb{N}} \subseteq C^{0, \alpha}(\bar{\Omega}) \text { is bounded. }
$$

We rewrite (35) as

$$
-\operatorname{div}\left(a(D y)-\hat{\gamma}_{n}\right)=0 \quad \text { on } \partial \Omega,\left.y\right|_{\partial \Omega}=0 .
$$

Then the nonlinear regularity theory of Lieberman [17] implies that we can find $\mu \in$ $(0,1)$ and $c_{20}>0$ such that

$$
y_{n} \in C_{0}^{1, \mu}(\bar{\Omega}) \quad \text { and } \quad\left\|y_{n}\right\|_{C_{0}^{1, \mu}(\bar{\Omega})} \leq c_{20} \quad \text { for all } n \in \mathbb{N} .
$$

From (37) and the compact embedding of $C_{0}^{1, \mu}(\bar{\Omega})$ into $C_{0}^{1}(\bar{\Omega})$, it follows that

$$
\left\{y_{n}\right\}_{n \in \mathbb{N}} \subseteq C_{0}^{1}(\bar{\Omega}) \text { is relatively compact. }
$$

Note that $\tau_{v_{n}}^{\lambda}(\cdot, u(\cdot)) \mapsto \tau_{v}^{\lambda}(\cdot, u(\cdot))$ in $L^{s}(\Omega)$. So, for the whole sequence, we have

$$
y_{n} \rightarrow u \quad \text { in } C_{0}^{1}(\bar{\Omega}) \text { as } n \rightarrow \infty .
$$

We set $y_{n}=y_{n}^{0}$ and consider the following Dirichlet problem:

$$
-\operatorname{div} a(D y(z))=\tau_{v_{n}}^{\lambda}\left(z, y_{n}^{0}(z)\right) \quad \text { in } \Omega,\left.y\right|_{\partial \Omega}=0 .
$$

As above this problem has a unique solution $y_{n}^{1} \in C_{0}^{1}(\bar{\Omega})$ and we have

$$
y_{n}^{1} \rightarrow u \quad \text { in } C_{0}^{1}(\bar{\Omega}) \text { as } n \rightarrow \infty .
$$


We continue this way and generate a sequence $\left\{y_{n}^{k}\right\}_{k, n \in \mathbb{N}} \subseteq C_{0}^{1}(\bar{\Omega})$ such that

$$
\begin{aligned}
& -\operatorname{div} a\left(D y_{n}^{k}(z)\right)=\tau_{v_{n}}^{\lambda}\left(z, y_{n}^{k-1}(z)\right) \quad \text { in } \Omega,\left.y_{n}^{k}\right|_{\partial \Omega}=0, \\
& y_{n}^{k} \rightarrow u \quad \text { in } C_{0}^{1}(\bar{\Omega}) \text { as } n \rightarrow \infty \text { for all } k \in \mathbb{N} .
\end{aligned}
$$

We will show that for every $n \in \mathbb{N}$

$$
\left\{y_{n}^{k}\right\}_{k \in \mathbb{N}} \subseteq W_{0}^{1, p}(\Omega) \text { is bounded }
$$

Arguing by contradiction, suppose that (40) is not true. By passing to a subsequence if necessary, we may assume that

$$
\left\|y_{n}^{k}\right\| \rightarrow \infty \quad \text { as } k \rightarrow \infty
$$

We set $w_{k}=\frac{y_{n}^{k}}{\left\|y_{n}^{k}\right\|}, k \in \mathbb{N}$. Then $\left\|w_{k}\right\|=1$ for all $k \in \mathbb{N}$ and so we may assume that

$$
w_{k} \stackrel{w}{\rightarrow} w \quad \text { in } W_{0}^{1, p}(\Omega) \text { and } w_{k} \rightarrow w \text { in } L^{p}(\Omega) \text { as } k \rightarrow \infty
$$

From (38) we have

$$
\begin{aligned}
& \int_{\Omega}\left(\frac{a\left(D y_{n}^{k}\right)}{\left\|y_{n}^{k}\right\| p^{p-1}}, D w_{k}\right)_{\mathbb{R}^{N}} d z=\int_{\Omega} \frac{\tau_{v_{n}}^{\lambda}\left(z, y_{n}^{k-1}\right)}{\left\|y_{n}^{k}\right\|^{p-1}} w_{k} d z \\
& \Rightarrow \quad \frac{c_{1}}{p-1}\left\|D w_{k}\right\|_{p}^{p} \leq \int_{\Omega} \frac{\tau_{v_{n}}^{\lambda}\left(z, y_{n}^{k-1}\right)}{\left\|y_{n}^{k}\right\|^{p-1}} w_{k} d z \quad \text { (see Lemma 2(b)). }
\end{aligned}
$$

On account of (24), (41) and (42), we have

$$
\begin{aligned}
& \int_{\Omega} \frac{\tau_{v_{n}}^{\lambda}\left(z, y_{n}^{k-1}\right)}{\left\|y_{n}^{k}\right\|^{p-1}} w_{k} d z \rightarrow 0 \quad \text { as } k \rightarrow \infty \\
& \quad \Rightarrow \quad w_{k} \rightarrow 0 \text { in } W_{0}^{1, p}(\Omega) \text { as } k \rightarrow \infty \quad(\text { see }(43)),
\end{aligned}
$$

which contradicts the fact that $\left\|w_{k}\right\|=1$ for all $k \in \mathbb{N}$.

Therefore (40) is true.

Then on account of (40) and the nonlinear regularity theory (see [17] and [21]), we infer that $\left\{y_{n}^{k}\right\}_{k \in \mathbb{N}} \subseteq C_{0}^{1}(\bar{\Omega})$ is relatively compact. Hence we may assume that

$$
y_{n}^{k} \rightarrow u_{n} \quad \text { in } C_{0}^{1}(\bar{\Omega}) \text { as } k \rightarrow \infty
$$

From (38) and hypothesis $\mathrm{H}_{1}(\mathrm{i})$, we have

$$
\begin{gathered}
-\operatorname{div} a\left(D u_{n}\right)=\tau_{v_{n}}^{\lambda}\left(z, u_{n}\right) \quad \text { in } \Omega,\left.u_{n}\right|_{\partial \Omega}=0, \\
\bar{u}_{\lambda} \leq u_{n} \leq \theta \quad \text { for all } n \in \mathbb{N}(\text { see }(24)) \\
\Rightarrow \quad u_{n} \in S_{v_{n}}^{\lambda} \quad \text { for all } n \in \mathbb{N} .
\end{gathered}
$$


From (45), (46) and the nonlinear regularity theory of Lieberman [17], it follows that $\left\{u_{n}\right\}_{n \in \mathbb{N}} \subseteq C_{0}^{1}(\bar{\Omega})$ is relatively compact. Then (39), (44) and the double limit lemma (see Gasinski-Papageorgiou [7], Problem 1.175, p. 61) imply that

$$
u_{n} \rightarrow u \quad \text { in } C_{0}^{1}(\bar{\Omega}) \text { with } u_{n} \in S_{v_{n}}^{\lambda} \text { for all } n \in \mathbb{N} \text {. }
$$

This proof is now complete.

Using this proposition, we can establish the compactness of the minimal solution map $\xi_{\lambda}(\cdot)$.

Proposition 10 If hypotheses $\mathrm{H}_{0}, \mathrm{H}_{1}$ hold and $\lambda \in\left(0, \hat{\lambda}_{*}\right]$, then the minimal solution map $\xi_{\lambda}: C_{0}^{1}(\bar{\Omega}) \mapsto C_{0}^{1}(\bar{\Omega})$ is compact.

Proof Let $B \subseteq C_{0}^{1}(\bar{\Omega})$ be bounded. Let $v \in B$ and $u=\xi_{\lambda}(v)$. We have

$$
\begin{aligned}
& -\operatorname{div} a(D u)=\lambda u^{-\eta}+f(z, u, D v) \quad \text { in } \Omega,\left.u\right|_{\partial \Omega}=0, \bar{u}_{\lambda} \leq u \leq \theta \\
& \Rightarrow \quad \frac{c_{1}}{p-1}\|D u\|_{p}^{p} \leq c_{21} \quad \text { for some } c_{21}>0 \\
& \Rightarrow \quad \xi_{\lambda}(B) \subseteq W_{0}^{1, p}(\Omega) \text { is bounded. }
\end{aligned}
$$

From this as before using the nonlinear regularity theory of Lieberman [17], we infer that

$$
\xi_{\lambda}(B) \subseteq C_{0}^{1}(\bar{\Omega}) \text { is relatively compact. }
$$

Next, we show that $\xi_{\lambda}(\cdot)$ is continuous. So, let $v_{n} \rightarrow v$ in $C_{0}^{1}(\bar{\Omega})$ and let $u_{n}=\xi_{\lambda}\left(v_{n}\right), n \in \mathbb{N}$ and $u=\xi_{\lambda}(v)$. From (47) we see that $\left\{u_{n}\right\}_{n \in \mathbb{N}} \subseteq C_{0}^{1}(\bar{\Omega})$ is relatively compact. Hence we may assume that

$$
u_{n} \rightarrow \widetilde{u} \quad \text { in } C_{0}^{1}(\bar{\Omega}) \text { as } n \rightarrow \infty
$$

Evidently $\tilde{u} \in S_{v}^{\lambda}$ and so we have

$$
u \leq \tilde{u}
$$

On the other hand, from Proposition 9, we know that we can find $\widetilde{u}_{n} \in S_{v_{n}}^{\lambda}, n \in \mathbb{N}$ such that

$$
\tilde{u}_{n} \rightarrow u \quad \text { in } C_{0}^{1}(\bar{\Omega}) \text { as } n \rightarrow \infty
$$

We have $u_{n} \leq \widetilde{u}_{n}$ for all $n \in \mathbb{N}$ and so from (48) and (50), we obtain

$$
\begin{aligned}
\tilde{u} & \leq u \\
& \Rightarrow \quad u=\widetilde{u} \quad(\operatorname{see}(49)) .
\end{aligned}
$$


So, for the whole sequence we have

$$
\begin{aligned}
u_{n} & \rightarrow u \quad \text { in } C_{0}^{1}(\bar{\Omega}) \\
& \Rightarrow \quad \xi_{\lambda}(\cdot) \text { is continuous } \\
& \Rightarrow \quad \xi_{\lambda}(\cdot) \text { is compact. }
\end{aligned}
$$

This proof is now complete.

Let $\mathcal{D}_{\lambda}=\left\{u \in C_{0}^{1}(\bar{\Omega}): u=t \xi_{\lambda}(u), 0<t<1\right\}$.

Proposition 11 If hypotheses $\mathrm{H}_{0}, \mathrm{H}_{1}$ hold and $\lambda \in\left(0, \hat{\lambda}_{*}\right]$, then $\mathcal{D}_{\lambda} \subseteq C_{0}^{1}(\bar{\Omega})$ is bounded.

Proof Let $u \in \mathcal{D}_{\lambda}$. We have

$$
\frac{1}{t} u=\xi_{\lambda}(u) \quad \text { with } 0<t<1 .
$$

It follows that

$$
\left\langle A\left(\frac{1}{t} u\right), h\right\rangle=\int_{\Omega}\left(\lambda\left(\frac{1}{t} u\right)^{-\eta}+f\left(z, \frac{1}{t} u, D u\right)\right) h d z
$$

for all $h \in W_{0}^{1, p}(\Omega)$.

From Proposition 7, we know that

$$
\begin{aligned}
\bar{u}_{\lambda} & \leq \frac{1}{t} u \\
& \Rightarrow \quad t \bar{u}_{\lambda} \leq u \leq \theta \quad(\text { since } t \in(0,1)) .
\end{aligned}
$$

In (51) we choose $h=u \in W_{0}^{1, p}(\Omega)$. Using Lemma 2(b), (52) (recall that $\bar{u}_{\lambda}^{-\eta} \in L^{s}(\Omega)$ ) and hypothesis $\mathrm{H}_{1}(\mathrm{i})$, we obtain

$$
\begin{aligned}
& \frac{c_{1}}{p-1}\|D u\|_{p}^{p} \leq c_{22}\left(1+\int_{\Omega}|f(z, u, D u)| d z\right) \text { for some } c_{22}>0 \\
& \leq c_{23}\left(1+\|D u\|^{p-1}\right) \text { for some } c_{23}>0 \\
& \Rightarrow \quad \mathcal{D}_{\lambda} \subseteq W_{0}^{1, p}(\Omega) \text { is bounded. }
\end{aligned}
$$

Then as before via the nonlinear regularity theory of Lieberman [17], we see that $\mathcal{D}_{\lambda} \subseteq$ $C_{0}^{1}(\bar{\Omega})$ is bounded (in fact relatively compact).

This proof is now complete.

So we can use Theorem 1 on the map $\xi_{\lambda}(\cdot)$ and produce a fixed point which is a positive solution of problem $\left(P_{\lambda}\right)$, for $\lambda \in\left(0, \hat{\lambda}_{*}\right]$. Concluding we can state the following existence theorem for problem $\left(P_{\lambda}\right)$.

Theorem 12 If hypotheses $\mathrm{H}_{0}, \mathrm{H}_{1}$ hold, then there exists $\hat{\lambda}_{*}>0$ such that for all $\lambda \in\left(0, \hat{\lambda}_{*}\right]$ problem $\left(P_{\lambda}\right)$ admits a positive solution

$\hat{u}_{\lambda} \in\left[\bar{u}_{\lambda}, \theta\right] \cap \operatorname{int} C_{+}$. 


\section{Funding}

This research is partially supported by the Fundamental Research Funds for the Central Universities of Central South University (No. 2019zzts211). This paper has been completed while Youpei Zhang was visiting University of Craiova (Romania) with the financial support of China Scholarship Council (No. 201906370079). Youpei Zhang would like to thank the China Scholarship Council and the Embassy of the People's Republic of China in Romania.

\section{Availability of data and materials}

This paper focuses on theoretical analysis, not involving experiments and data.

\section{Competing interests}

The authors declare that they have no competing interests.

\section{Authors' contributions}

The authors have equal contributions to each part of this paper. All authors read and approved the final manuscript.

\section{Author details}

${ }^{1}$ Department of Mathematics, National Technical University, Zografou Campus, Athens 15780, Greece. ${ }^{2}$ School of Mathematics and Statistics, Central South University, Changsha, Hunan 410083, China. ${ }^{3}$ Department of Mathematics, University of Craiova, Street A.I. Cuza No. 13, 200585 Craiova, Romania.

\section{Publisher's Note}

Springer Nature remains neutral with regard to jurisdictional claims in published maps and institutional affiliations.

Received: 26 August 2020 Accepted: 16 September 2020 Published online: 23 September 2020

\section{References}

1. Bai, Y.: Existence of solutions to nonhomogeneous Dirichlet problems with dependence on the gradient. Electron. J. Differ. Equ. 2018, 101 (2018)

2. Bai, Y., Gasinski, L., Papageorgiou, N.S.: Nonlinear nonhomogeneous Robin problems with dependence on the gradient. Bound. Value Probl. 2018, 17 (2018)

3. Bobkov, V., Tanaka, M.: On sign-changing solutions for $(p, q)$-Laplace equations with two parameters. Adv. Nonlinear Anal. 8, 101-129 (2019)

4. Candito, P., Gasinski, L., Papageorgiou, N.S.: Nonlinear nonhomogeneous Robin problems with convection. Ann Acad. Sci. Fenn., Math. 44, 755-767 (2019)

5. Faraci, F., Motreanu, D., Puglisi, D.: Positive solutions of quasi-linear elliptic equations with dependence on the gradient. Calc. Var. Partial Differ. Equ. 54, 525-538 (2015)

6. Gasinski, L., Krech, I., Papageorgiou, N.S.: Nonlinear nonhomogeneous Robin problems with gradient dependent reaction. Nonlinear Anal., Real World Appl. 55, 103135 (2020)

7. Gasinski, L., Papageorgiou, N.S.: Exercises in Analysis. Part 1. Springer, Cham (2014)

8. Gasinski, L., Papageorgiou, N.S.: Nonlinear Analysis. Chapman \& Hall/CRC, Boca Raton (2016)

9. Gasinski, L., Papageorgiou, N.S.: Exercises in Analysis. Part 2: Nonlinear Analysis. Springer, Cham (2016)

10. Gasinski, L., Papageorgiou, N.S.: Positive solutions for nonlinear elliptic problems with dependence on the gradient J. Differ. Equ. 263, 1451-1476 (2017)

11. Gasinski, L., Winkert, P.: Existence and uniqueness results for double phase problems with convection term. J. Differ Equ. 268, 4183-4193 (2020)

12. Gilbarg, D., Trudinger, N.S.: Elliptic Partial Differential Equations of Second Order, 2nd edn. Springer, Berlin (1998)

13. Guo, Z., Webb, J.R.L.: Uniqueness of positive solutions for quasilinear elliptic equations when a parameter is large. Proc. R. Soc. Edinb., Sect. A 124, 189-198 (1994)

14. Hu, S., Papageorgiou, N.S.: Handbook of Multivalued Analysis. Volume I: Theory. Kluwer Academic, Dordrecht (1997)

15. Ladyzhenskaya, O.A., Uraltseva, N.N.: Linear and Quasilinear Elliptic Equations. Academic Press, New York (1968)

16. Lazer, A.C., McKenna, P.J.: On a singular nonlinear elliptic boundary value problem. Proc. Am. Math. Soc. 111, 721-730 (1991)

17. Lieberman, G.: The natural generalization of the natural conditions of Ladyzhenskaya and Ural'tseva for elliptic equations. Commun. Partial Differ. Equ. 16, 311-361 (1991)

18. Liu, Z., Papageorgiou, N.S.: Positive solutions for resonant $(p, q)$-equations with convection. Adv. Nonlinear Anal. 10, 217-232 (2021)

19. Papageorgiou, N.S., Rădulescu, V.D.: Coercive and noncoercive nonlinear Neumann problems with indefinite potential. Forum Math. 28, 545-571 (2016)

20. Papageorgiou, N.S., Rădulescu, V.D., Repovš, D.D.: Positive solutions for nonvariational Robin problems. Asymptot. Anal. 108, 243-255 (2018)

21. Papageorgiou, N.S., Rădulescu, V.D., Repovš, D.D.: Nonlinear Analysis-Theory and Methods. Springer, Cham (2019)

22. Papageorgiou, N.S., Rădulescu, V.D., Repovš, D.D.: Positive solutions for nonlinear Neumann problems with singular terms and convection. J. Math. Pures Appl. 136, 1-21 (2020)

23. Papageorgiou, N.S., Rădulescu, V.D., Repovš, D.D.: Nonlinear nonhomogeneous singular problems. Calc. Var. Partial Differ. Equ. 59, 9 (2020)

24. Papageorgiou, N.S., Vetro, C., Vetro, F.: Existence of positive solutions for nonlinear Dirichlet problems with gradient dependence and arbitrary growth. Electron. J. Qual. Theory Differ. Equ. 18, 9 (2018)

25. Papageorgiou, N.S., Winkert, P.: Singular p-Laplacian equations with superlinear perturbation. J. Differ. Equ. 266 1462-1487 (2019)

26. Papageorgiou, N.S., Zhang, C.: Noncoercive resonant $(p, 2)$-equations with concave terms. Adv. Nonlinear Anal. 9 228-249 (2020) 
27. Papageorgiou, N.S., Zhang, Y.: Constant sign and nodal solutions for superlinear $(p, q)$-equations with indefinite potential and concave boundary term. Adv. Nonlinear Anal. 10, 76-101 (2021)

28. Pucci, P., Serrin, J.: The Maximum Principle. Progress in Nonlinear Differential Equations and Their Applications, vol. 73. Birkhäuser, Basel (2007)

29. Rădulescu, V.D.: Isotropic and anisotropic double-phase problems: old and new. Opusc. Math. 39, 259-279 (2019)

30. Ragusa, M.A., Tachikawa, A.: Regularity for minimizers for functionals of double phase with variable exponents. Adv. Nonlinear Anal. 9, 710-728 (2020)

31. Tanaka, M.: Existence of positive solution for quasilinear elliptic equations with nonlinearity including the gradient. Bound. Value Probl. 2013, 173 (2013)

Submit your manuscript to a SpringerOpen ${ }^{\circ}$ journal and benefit from:

- Convenient online submission

- Rigorous peer review

- Open access: articles freely available online

- High visibility within the field

- Retaining the copyright to your article

Submit your next manuscript at $\gg$ springeropen.com 